\title{
Sleep Disturbance Severity is Associated With Cognitive Impairment in Patients With First-episode, Treatment-naïve Major Depressive Disorders
}

\section{Feihuan Cui}

Capital Medical University Affiliated Anzhen Hospital

Qi Liu ( $D$ liu_qee@sina.com )

Peking University Sixth Hospital, Peking University Institute of Mental Health, Beijing, China

Xiaozhen Lv

Peking University Sixth Hospital

Rainer Leonhart

University of Freiburg,Institute for Psychology

Hongjun Tian

Nankai University Affiliated Anding Hopital

Jing Wei

Peking Union Medical College Hospital

Kerang Zhang

First Hospital of Shanxi Medical University

Gang Zhu

First Affiliated Hospital of China Medical University

Qiaoling Chen

Dalian Seventh People's Hospital

\section{Gang Wang}

Beijing Anding Hospital

\section{Xueyi Wang}

Hebei Medical University First Affiliated Hospital

\section{Nan Zhang}

Tianjin Medical University General Hospital

\section{Yu Huang}

National Engineering Research Center for Software Engineering, Peiking University

\section{Tianmei Si}

Peking University Sixth Hospital

\section{Xin Yu}

Peking University Sixth Hospital 


\section{Research article}

Keywords: major depressive disorder, cognitive functioning, sleep disorders, executive functioning, information processing speed

Posted Date: August 7th, 2020

DOI: https://doi.org/10.21203/rs.3.rs-52088/v1

License: (c) (i) This work is licensed under a Creative Commons Attribution 4.0 International License.

Read Full License

Version of Record: A version of this preprint was published at BMC Psychiatry on April 19th, 2021. See the published version at https://doi.org/10.1186/s12888-021-03194-2. 


\section{Abstract}

\section{Background}

Sleep disorders and cognitive impairment are common in patients with major depressive disorder (MDD), though the relationship between the two remains unclear. We investigated this association in firstepisode, treatment-naïve patients with MDD.

\section{Method}

We analyzed data from 242 patients withMDD. We divided the patients into 2 groups based on sleep disturbance severity and compared the cognitive impairment odds ratios in different domains between the groups.

\section{Results}

A total of 121 pairs of patients were matched (age39.4 $\pm 10.1,70.2 \%$ female). After propensity score matching, the odds ratios for cognitive impairment in patients with MDD and serious sleep disorders were 1.922 (1.068-3.459, $P=0.029)$ in executive functioning; $2.023(1.211-3.379, P=0.007)$ in executive shifting; $1.749(1.000-3.060, P=0.050)$ in executive inhibition; and $1.813(1.038-3.167, P=0.037)$ in information processing speed.

\section{Limitations}

The cross-sectional data on the relationship between sleep disorders and cognitive impairment were weak. Our study population was restricted to Chinese adults aged 18 to 65 years, so the results may not be generalizable to other populations.Because our sample size was limited, some variables were not included in the study.

\section{Conclusions}

Sleep disturbance is associated with cognitive impairment in the domains of executive functioning and information processing speed in first-episode, treatment-naïve patients with MDD. Sleep problems can be important symptoms for physicians in clinical practice and can aid in recognizing cognitive impairment in patients with MDD. Further study is required to confirm our results.

\section{Trial registration}

ClinicalTrials.gov: NCT02023567; registration date: December 2013.

\section{Background}

Major depressive disorder (MDD) is a disabling disease with significant social and economic consequences, including decreased work productivity and poor psychosocial outcomes [1, 2]. MDD has 
been associated with cognitive impairment in several domains, including attention, memory, executive functioning, and information processing speed [3], and approximately $90 \%$ of patients with MDD complain about impaired cognition [4].

Patients with first-episode MDD can suffer from impaired cognition [5]. Cognitive dysfunction is associated with a high risk of relapse and a low rate of remission [6, 7], increasing the risk of treatmentresistant MDD, so it is imperative to intervene early during the course of the first episode of MDD. Treatment should not just focus on the remission of affective symptoms but also on improving the patient's quality of life and recovering social functioning $[8,9]$. Cognitive deficits are barriers to full resolution of depressive symptoms and restoration of social functioning, so cognition is an important treatment target in patients with MDD.

Sleep disturbances are common complaints in patients who are depressed [10], and they, in turn, increase the risk of developing a depressive disorder $[11,12]$. Sleep disorders are manifest in different ways, including difficulty falling asleep, frequent nocturnal awakenings, and waking early in the morning, unable to go back to sleep. Several studies have explored the relationship between sleep and cognitive functioning $[13,14]$, and a recent meta-analysis concluded that insomnia has a profound effect on a wide range of cognitive domains [15].

Researchers have found several risk factors for cognitive impairment in patients with MDD, including age [16], age of onset [17], depression severity and duration, comorbid physical disease, episode frequency [1], childhood trauma $[18,19]$, and MDD subtype [20]. Most studies have focused on recurrent depressive disorders or cognitive impairment in late-onset depression [21]. Some risk factors are unmodifiable, such as age, and others are potentially modifiable, such as MDD severity. Though previous studies have explored the negative consequences of sleep disturbances in patients with MDD or cognitive impairment $[12,22,23]$, it is still not known about the role, if any, of sleep disturbances play in the development of cognitive impairment in patients with MDD.

Previous studies have included patients with both first-episode and recurrent depressive disorders, and the influence of antidepressants has not been clarified. So, we aimed to examine whether sleep disturbance severity can be a risk factor for cognitive impairment in unmedicated patients with MDD. If so, then treating sleep disturbances may be an option in reducing cognitive impairment in a clinical setting. Here we report on the relationship between sleep disturbances and cognitive impairment in the setting of a clinical trial.

\section{Method}

\section{Participants}

Between December 2013 and December 2016, researchers recruited Chinese adults to participate in the multicenter, multistage, prospective study Objective Diagnostic Indicators and Individualized Drug Intervention of Major Depressive Disorder (OIMDD), project No. 2013CB531305. We conducted a 
secondary, cross-sectional analysis using the baseline data from OIMDD for participants diagnosed with MDD. Nine hospitals in China participated in the study. All participants provided written consent, and the study protocol was approved by the research ethics board of the institution where it was performed.

Study participants included patients between the ages of 18 and 65 years who had been diagnosed with their first episode of MDD, did not receive any antidepressant treatment in the acute phase of the disease, and had a total score of $\geq 14$ on the 17-item Hamilton Rating Scale for Depression (HAMD-17) [24]. Patients were excluded if they had severe somatic diseases, such as severe heart disease, malignant tumors, or a history of epilepsy. Pregnant or lactating women were also excluded [25].

\section{Depression Diagnosis and Clinical Assessment}

Using criteria from the Diagnostic and Statistical Manual of Mental Disorders, Fourth Edition (DSM-IV), psychiatrists made a diagnosis of MDD and then conducted structured clinical interviews with the prospective participants using the Mini-International Neuropsychiatric Interview (MINI), Chinese Version 5.0.0 [26]. After the interviews, HAMD-17 was used to assess depressive symptoms, evaluating functioning in 5 subscales: cognitive impairment, retardation, anxiety or somatization, sleep disturbance, and weight change [27]. Sleep disturbance severity was assessed by evaluating the followingmetrics on the HAMD-17 scale: item 4, difficulty falling asleep; item 5, waking in the middle of the night for any reason except to void; and item 6 , waking early in the morning and unable to go back to sleep. Each item could be rated from 0 (no difficulty) to 2 (nightly difficulty), with a total possible score of 6 . A sleep subscale score $>4$ was defined as a serious sleep disorder, and patients in this category were assigned into Group 1; the remainder, were assigned into Group 2. The Hamilton Anxiety Rating Scale (HAMA) was used to assess 2 areas of somatic anxiety: muscular (pains and aches, twitching, stiffness, myoclonic jerks, grinding of teeth, unsteady voice, and increased muscular tone)and sensory (tinnitus, blurring of vision, hot and cold flushes, feelings of weakness, and pricking sensation) $[28,29]$.

\section{Cognitive Tests}

A battery of cognitive tests were performed to evaluate 5 cognitive domains: attention (vigilance), assessed by the Continuous Performance Test (CPT); speed of information processing, assessed by the Animal Verbal Fluency Scale (AVFS), Digit Symbol Coding Test (DSCT), and Color Trial Test (CTT); learning, assessed by the immediate recall of Brief Visual Memory Test-Revised (BVMT-R) and Hopkins Verbal Learning Test-Revised (HVLT-R); memory, assessed by delayed recall of Brief Visual Memory TestRevised (BVMT-R) and Hopkins Verbal Learning Test-Revised (HVLT-R); executive functioning,assessed by the Stroop Color Word Test (SCWT, which assessed executive inhibition) and color line II (which assessed executive-shifting). We selected the cognitive test battery in our study from the MCCB (MATRICS Consensus Cognitive Battery) of the MATRICS (the Measurement and Treatment Research to Improve Cognition in Schizophrenia) study. The MCCB has been widely used to evaluate the cognitive function of

patients with mental disorders. The test scores for each domain were transferred into global deficit scores (GDS), which were adjusted for age, sex, and education level. A global deficit score $\geq 0.5$ was defined as cognitive impairment [30,31]. 


\section{Confounding Covariables}

In addition to gathering basic demographic data-marital status, living situation (whether the subject lives alone or with others), religious affiliation, work status, the type of work (whether it requires ental labor, physical labor, or both), independence (degree to which the subject depends on someone else for life's basics), and body mass index (BMI), we obtained information on potential confounding covariables that could alter a person's risk for cognitive impairment. Some comorbid conditions are known to increase the risk of cognitive impairment, including cardiovascular disease and diabetes. Other suspected risk factors include duration of disorder, severity of depression and/or anxiety, family history of psychiatric disorders, history of alcohol abuse, smoking history, and childhood trauma[1].

We collected previous disease history at baseline, including hyperlipidemia, hypertension, and diabetes. Diabetes was defined as a fasting blood glucose (FBG) level $\geq 126 \mathrm{mg} / \mathrm{dL}(7.0 \mathrm{mmol} / \mathrm{L})$, oral glucose tolerance test $\geq 200 \mathrm{mg} / \mathrm{dL}$ ( $11.1 \mathrm{mmol} / \mathrm{L}), \mathrm{HbA} 1 \mathrm{c} \geq 48 \mathrm{mmol} / \mathrm{L}(6.5 \%)$, or a history of diabetes mellitus. Hyperlipidemia was defined as having total cholesterol $(T C) \geq 5.17 \mathrm{mmol} / \mathrm{L}$, triglycerides $(T G) \geq 1.7$ $\mathrm{mmol} / \mathrm{L}$, low-density lipoprotein (LDL) cholesterol $\geq 3.37 \mathrm{mmol} / \mathrm{L}$, or a history of hyperlipidemia. Social demographic variables were assessed using questionnaires that asked about age, sex, marital status, occupation, alcohol use, and smoking status. Body mass index was calculated as weight in kilograms divided by squared height in meters $\left(\mathrm{kg} / \mathrm{m}^{2}\right)$. BMl was divided into low weight $(\mathrm{BMl}<18.5)$, normal weight ( $\mathrm{BMI} \geq 18.5$ to $<24)$, overweight (BMI $>24$ to $\leq 28)$, and obesity ( $\mathrm{BMI}>28)$.

Childhood trauma was assessed using the childhood trauma questionnaire (CTQ), which evaluates the subject's experience of abuse and/or neglect before the age of 18 years. The CTQ includes domains of emotional, physical, and sexual abuse, as well as of emotional and physical neglect. Items are rated from 1 ("never true") to 5 ("very often true") according to the frequency with which each event occurred in childhood. We considered the history of trauma as a dichotomic variable (yes/no) if the person rated items as moderate or severe according to the subscale cutoff criteria in at least one type of trauma [32].

\section{Statistical Analyses}

Summary statistics were presented as mean \pm standard deviation for variables that conformed to a normal distribution, as medians and quartile for data did not conform to a normal distribution, and as percentages for categorical variables. To make the 2 groups more comparable, we performed propensity score matching.Propensity scores were estimated using a logistic regression model that contained known or suspected covariates that were unbalanced between the 2 groups. To perform the propensity score matching, we included the type of work, marital status, religion, alcohol use, and HAMD-17 weight subscale. Because the independent-variable global deficit scores are functions converted fromstandard scores corrected by age, sex, and education, we did not include these variables in the propensity score matching. Subjects were matched 1:1 without replacement, using a 0.00 caliper width. Effect size was calculated to estimate the balance of the baseline data between the 2 groups. Cohen $d$ was calculated 
using a t test and $\varphi$ (phi) or $\varphi c$ (Cramer's phi) was calculated using the chi-square test. Effect size $>0.20$ was considered to be an imbalance between the 2 groups.

Statistical analyses were conducted using SPSS 25.0 (SPSS Inc, Chicago, Illinois, USA) and version R 3.3.3(Foundation for Statistical Computing,Vienna, Austria). The R package of Matchlt was used forthe propensity score analysis. Logistic regression analysis was used to estimate the odds ratios for the global deficit scores for each cognitive domain and their corresponding $95 \%$ confidence intervals (Cls).

\section{Results}

Of the 296 patients included in our analysis, 121 had symptoms of serious sleep disorders. Chi-square analysis and nonparametric test of the demographic and clinical characteristics between the 2 groups revealed significant differences in the groups' social demographics (see Table 1), including type of work (10.924, $P=0.004)$, marital status $(17.072, P<0.001)$, living situation $(19.919, P<0.001)$, religion (4.457, $P=0.035)$, alcohol use $(14.284, P=0.001)$, and HAMD weight subscale $(P=0.001)$. Because the 2 groups were so heterogeneous, we performed propensity score matching and the variables above were included into matching, after which the baseline characteristics of the 2 groups were highly comparable and we were able to successfully match 121 pairs of participants (average age $39.42 \pm 10.07,70.2 \%$ female). No differences in the other variables were observed between the 2 groups, with the exception of age (Table 2). The effects of sleep symptom severity between the 2 groups were similar before and after propensity score matching, with the exception of word fluency (Tables 3 and 4). 
Table 1

Demographic and clinical characteristics of study participants before matching.

\begin{tabular}{|c|c|c|c|c|c|}
\hline & $\begin{array}{l}\text { Group } 1 \\
(121)\end{array}$ & $\begin{array}{l}\text { Group } 2 \\
(175)\end{array}$ & $\begin{array}{l}\text { Effect } \\
\text { Size }\end{array}$ & $\begin{array}{l}\mathrm{t}, \mathrm{z} \text {, or Chi- } \\
\text { square }\end{array}$ & $\begin{array}{l}P \\
\text { value }\end{array}$ \\
\hline Age & $41.8 \pm 9.2$ & $35.1 \pm 10.6$ & 0.675 & $-5.600^{a}$ & $\begin{array}{l}< \\
0.001\end{array}$ \\
\hline Education years (year) & $11.9 \pm 4.0$ & $13.1 \pm 3.7$ & 0.311 & $2.588^{a}$ & 0.010 \\
\hline Sex (male) (\%) & $39(32.2)$ & $52(29.7)$ & 0.027 & $0.213^{b}$ & 0.645 \\
\hline Occupation (\%) & $83(68.6)$ & $127(72.6)$ & 0.043 & $0.549^{b}$ & 0.459 \\
\hline Full-time or part-time & $38(31.4)$ & $48(27.4)$ & & & \\
\hline \multicolumn{6}{|l|}{ Unemployed or retired } \\
\hline Nature of work (\%) & $58(47.9)$ & $117(66.9)$ & \multirow[t]{4}{*}{0.192} & \multirow[t]{4}{*}{$10.924^{b}$} & \multirow[t]{4}{*}{0.004} \\
\hline Mental talents labor & $39(32.2)$ & $33(18.9)$ & & & \\
\hline Physical labor & $24(19.9)$ & $25(14.3)$ & & & \\
\hline \multicolumn{3}{|l|}{ Both } & & & \\
\hline Marital status (\%) & $101(83.5)$ & $107(61.1)$ & \multirow[t]{3}{*}{0.240} & \multirow[t]{3}{*}{$17.072^{b}$} & \multirow{3}{*}{$\dot{0} 001$} \\
\hline Married or remarried & $20(16.5)$ & $68(38.9)$ & & & \\
\hline \multicolumn{3}{|l|}{$\begin{array}{l}\text { Divorced or widowed or } \\
\text { single }\end{array}$} & & & \\
\hline Living situation (\%) & $12(9.9)$ & $15(8.6)$ & \multirow[t]{5}{*}{0.259} & \multirow[t]{5}{*}{$19.919^{b}$} & \multirow{5}{*}{$<.001$} \\
\hline Living alone & $31(25.6)$ & $66(37.7)$ & & & \\
\hline With parents or spouse & $9(7.4)$ & $35(20.0)$ & & & \\
\hline With spouse and child & $69(57.1)$ & $59(33.7)$ & & & \\
\hline \multicolumn{3}{|l|}{ Other } & & & \\
\hline Independence (\%) & $115(95.0)$ & $156(89.1)$ & 0.104 & $3.219^{b}$ & 0.073 \\
\hline Independent & $6(5.0)$ & 19 (10.9) & & & \\
\hline \multicolumn{6}{|l|}{ Need assistance } \\
\hline Childhood trauma (\%) & $39(32.2)$ & $71(40.6)$ & 0.085 & $2.131^{b}$ & 0.144 \\
\hline \multicolumn{6}{|l|}{ Yes } \\
\hline \multicolumn{6}{|c|}{$\begin{array}{l}\text { G1 = Group 1, patients with serious sleep symptoms; G2 = Group 2, patients without serious sleep } \\
\text { symptoms. }\end{array}$} \\
\hline a Independent sample t & hi-square & Fisher's ex & ${ }^{t} \cdot d n$ & metric test. & \\
\hline
\end{tabular}




\begin{tabular}{|c|c|c|c|c|c|}
\hline & $\begin{array}{l}\text { Group } 1 \\
(121)\end{array}$ & $\begin{array}{l}\text { Group } 2 \\
\text { (175) }\end{array}$ & $\begin{array}{l}\text { Effect } \\
\text { Size }\end{array}$ & $\begin{array}{l}\mathrm{t}, \mathrm{z} \text {, or Chi- } \\
\text { square }\end{array}$ & $\begin{array}{l}P \\
\text { value }\end{array}$ \\
\hline Religion (\%) & $117(96.7)$ & $158(90.3)$ & 0.123 & $4.457^{b}$ & 0.035 \\
\hline \multicolumn{6}{|l|}{ No } \\
\hline Smoking history (\%) & 89 (73.6) & $136(77.7)$ & 0.105 & $3.246^{b}$ & 0.197 \\
\hline Never & $11(9.0)$ & $7(4.0)$ & & & \\
\hline Quit smoking & $21(17.4)$ & 32 (18.3) & & & \\
\hline \multicolumn{6}{|l|}{ Smoking now } \\
\hline Alcohol use (\%) & $89(73.6)$ & $106(60.6)$ & 0.220 & $14.284^{c}$ & 0.001 \\
\hline Never & $10(8.3)$ & $5(2.8)$ & & & \\
\hline Quit drinking & $22(18.1)$ & $64(36.6)$ & & & \\
\hline \multicolumn{6}{|l|}{ Drinking now } \\
\hline Hypertension (\%) & $14(11.6)$ & $10(5.7)$ & 0.105 & $3.293^{b}$ & 0.070 \\
\hline \multicolumn{6}{|l|}{ Yes } \\
\hline Diabetes (\%) & $7(5.8)$ & $4(2.3)$ & 0.091 & $2.448^{c}$ & 0.131 \\
\hline \multicolumn{6}{|l|}{ Yes } \\
\hline Hyperlipidemia (\%) & $9(7.4)$ & 18 (10.3) & 0.049 & $0.700^{b}$ & 0.403 \\
\hline \multicolumn{6}{|l|}{ Yes } \\
\hline First degree relatives (\%) & $20(16.5)$ & $33(18.9)$ & 0.030 & $0.264^{b}$ & 0.608 \\
\hline \multicolumn{6}{|l|}{ Yes } \\
\hline Duration of MDD (months) & $\begin{array}{l}6.0(2.0 \\
12.0)\end{array}$ & $\begin{array}{l}6.0(3.0, \\
21.0)\end{array}$ & & $-1.513^{d}$ & 0.130 \\
\hline BMI (\%) & $11(9.1)$ & $18(10.3)$ & 0.144 & $6.157^{b}$ & 0.104 \\
\hline Low weight & $59(48.8)$ & 107 (61.1) & & & \\
\hline Normal & $41(33.8)$ & $42(24.0)$ & & & \\
\hline Overweight & $10(8.3)$ & $8(4.6)$ & & & \\
\hline \multicolumn{6}{|l|}{ Obesity } \\
\hline HAMD anxiety subscale & $5.8 \pm 2.2$ & $6.1 \pm 1.8$ & 0.149 & $0.972^{a}$ & 0.332 \\
\hline \multicolumn{6}{|c|}{$\begin{array}{l}\text { G1 = Group 1, patients with serious sleep symptoms; G2 = Group 2, patients without serious sleep } \\
\text { symptoms. }\end{array}$} \\
\hline
\end{tabular}




\begin{tabular}{|c|c|c|c|c|c|}
\hline & $\begin{array}{l}\text { Group } 1 \\
(121)\end{array}$ & $\begin{array}{l}\text { Group } 2 \\
(175)\end{array}$ & $\begin{array}{l}\text { Effect } \\
\text { Size }\end{array}$ & $\begin{array}{l}\mathrm{t}, \mathrm{z} \text {, or Chi- } \\
\text { square }\end{array}$ & $\begin{array}{l}P \\
\text { value }\end{array}$ \\
\hline HAMD weight subscale & $0.0(0.0,2.0)$ & $0.0(0.0,1.0)$ & & $-3.422^{d}$ & 0.001 \\
\hline HAMD cognition subscale & $3.0 \pm 1.9$ & $3.1 \pm 1.6$ & 0.057 & $0.493^{a}$ & 0.623 \\
\hline HAMD retard subscale & $6.8 \pm 1.7$ & $6.5 \pm 1.8$ & 0.171 & $-1.534^{a}$ & 0.126 \\
\hline HAMA somatic anxiety & $13.4 \pm 4.1$ & $13.2 \pm 3.7$ & 0.051 & $-0.544^{a}$ & 0.687 \\
\hline $\begin{array}{l}\text { HAMA psychological } \\
\text { anxiety }\end{array}$ & $6.9 \pm 4.3$ & $7.5 \pm 4.2$ & 0.141 & $1.182^{a}$ & 0.238 \\
\hline \multicolumn{6}{|c|}{$\begin{array}{l}\text { G1 = Group 1, patients with serious sleep symptoms; G2 = Group 2, patients without serious sleep } \\
\text { symptoms. }\end{array}$} \\
\hline \multicolumn{6}{|c|}{ a Independent sample t-test; ${ }^{\mathrm{b}}$ chi-square test; ${ }^{\mathrm{c}}$ Fisher's exact test; ${ }^{\mathrm{d}}$ nonparametric test. } \\
\hline
\end{tabular}


Table 2

Demographic and clinical characteristics after matching.

\begin{tabular}{|c|c|c|c|c|c|}
\hline & $\begin{array}{l}\text { Group } 1 \\
\text { (121) }\end{array}$ & $\begin{array}{l}\text { Group } 2 \\
\text { (121) }\end{array}$ & $\begin{array}{l}\text { Effect } \\
\text { Size }\end{array}$ & $\begin{array}{l}t, z \text {, or Chi- } \\
\text { square }\end{array}$ & $\begin{array}{l}P \\
\text { Value }\end{array}$ \\
\hline Age & $41.8 \pm 9.2$ & $37.1 \pm 10.4$ & 0.478 & -3.699 a & $\begin{array}{l}< \\
0.001\end{array}$ \\
\hline Education years & $11.9 \pm 4.0$ & $12.7 \pm 3.8$ & 0.205 & $1.588^{a}$ & 0.114 \\
\hline Sex (male) (\%) & 39 (32.2) & $33(27.3)$ & 0.054 & $0.712^{b}$ & 0.399 \\
\hline Occupation (\%) & $83(68.6)$ & $86(71.1)$ & 0.027 & $0.177^{\mathrm{b}}$ & 0.674 \\
\hline $\begin{array}{l}\text { Full-time or part-time } \\
\text { Unemployed or retired }\end{array}$ & 38 (31.4) & $35(28.9)$ & \multicolumn{2}{|c|}{ Unemployed or retired } & \\
\hline Nature of work (\%) & $58(48.0)$ & $75(62.0)$ & 0.141 & $4.836^{b}$ & 0.089 \\
\hline Mental talents labor & $39(32.2)$ & $28(23.1)$ & & & \\
\hline Physical labor & $24(19.8)$ & $18(14.9)$ & & & \\
\hline \multicolumn{6}{|l|}{ Both } \\
\hline Marital status (\%) & 101 (83.5) & $89(73.6)$ & 0.121 & $3.527^{b}$ & 0.060 \\
\hline \multicolumn{6}{|l|}{$\begin{array}{l}\text { Divorced, widowed, or } \\
\text { single }\end{array}$} \\
\hline Living situation (\%) & $12(9.9)$ & $9(7.4)$ & 0.166 & $6.646^{b}$ & 0.084 \\
\hline Living alone & $31(25.6)$ & $42(34.7)$ & & & \\
\hline With parents or spouse & $9(7.4)$ & $17(14.1)$ & & & \\
\hline $\begin{array}{l}\text { With spouse and child } \\
\text { Other }\end{array}$ & $69(57.1)$ & $53(43.8)$ & & & \\
\hline Independence (\%) & $115(95.0)$ & $112(92.6)$ & 0.051 & $0.640^{b}$ & 0.424 \\
\hline \multicolumn{6}{|l|}{ Need assistance } \\
\hline \multicolumn{5}{|l|}{ Yes } & 0.346 \\
\hline \multicolumn{6}{|c|}{$\begin{array}{l}\text { G1 = Group 1, patients with serious sleep symptoms; G2 = Group 2, patients without serious sleep } \\
\text { symptoms. }\end{array}$} \\
\hline a Independent sample t- & hi-square & risnterse & est; ${ }^{d} n$ & metric test. & \\
\hline
\end{tabular}




\begin{tabular}{|c|c|c|c|c|c|}
\hline & $\begin{array}{l}\text { Group } 1 \\
\text { (121) }\end{array}$ & $\begin{array}{l}\text { Group } 2 \\
(121)\end{array}$ & $\begin{array}{l}\text { Effect } \\
\text { Size }\end{array}$ & $\begin{array}{l}\mathrm{t}, \mathrm{z} \text {, or Chi- } \\
\text { square }\end{array}$ & $\stackrel{P}{\text { Value }}$ \\
\hline Religion (\%) & 117 (96.7) & $114(94.2)$ & 0.061 & $0.857^{b}$ & 0.355 \\
\hline \multicolumn{6}{|l|}{ No } \\
\hline Smoking (\%) & $89(73.6)$ & 96 (79.3) & 0.102 & $2.539^{c}$ & 0.281 \\
\hline Never & $11(9.0)$ & $5(4.1)$ & & & \\
\hline Quit smoking & $21(17.4)$ & $20(16.5)$ & & & \\
\hline \multicolumn{6}{|l|}{ Smoking now } \\
\hline Alcohol use (\%) & 89 (73.6) & $84(69.4)$ & 0.143 & $4.916^{c}$ & 0.086 \\
\hline Never & $10(8.2)$ & $4(3.3)$ & & & \\
\hline Quit drinking & $22(18.2)$ & 33 (27.3) & & & \\
\hline \multicolumn{6}{|l|}{ Drinking now } \\
\hline Hypertension (\%) & $14(11.6)$ & $9(7.4)$ & 0.105 & $1.201^{b}$ & 0.381 \\
\hline \multicolumn{6}{|l|}{ Yes } \\
\hline Diabetes (\%) & $7(5.8)$ & $3(2.5)$ & 0.091 & $1.669^{c}$ & 0.333 \\
\hline \multicolumn{6}{|l|}{ Yes } \\
\hline Hyperlipidemia (\%) & $9(7.4)$ & $13(10.7)$ & 0.049 & $0.800^{b}$ & 0.371 \\
\hline \multicolumn{6}{|l|}{ Yes } \\
\hline $\begin{array}{l}\text { First degree of relatives } \\
(\%)\end{array}$ & $20(16.5)$ & $20(16.5)$ & 0.030 & $0.000^{b}$ & 1.000 \\
\hline \multicolumn{6}{|l|}{ Yes } \\
\hline $\begin{array}{l}\text { Duration of MDD } \\
\text { (months) }\end{array}$ & $\begin{array}{l}6.0(2.0 \\
12.0)\end{array}$ & $\begin{array}{l}6.0(3.0 \\
12.5)\end{array}$ & & $0.198^{d}$ & 0.843 \\
\hline BMI (\%) & $11(9.1)$ & $13(10.7)$ & 0.139 & $4.709^{b}$ & 0.194 \\
\hline Low weight & $59(48.8)$ & $73(60.3)$ & & & \\
\hline Normal & $41(33.8)$ & $29(24.0)$ & & & \\
\hline Overweight & $10(8.3)$ & $6(5.0)$ & & & \\
\hline Obesity & & & & & \\
\hline
\end{tabular}

G1 = Group 1, patients with serious sleep symptoms; G2 = Group 2, patients without serious sleep symptoms.

${ }^{a}$ Independent sample t-test; ${ }^{b}$ chi-square test; ${ }^{c}$ Fisher's exact test; ${ }^{d}$ nonparametric test. 


\begin{tabular}{|c|c|c|c|c|c|}
\hline & $\begin{array}{l}\text { Group } 1 \\
\text { (121) }\end{array}$ & $\begin{array}{l}\text { Group } 2 \\
(121)\end{array}$ & $\begin{array}{l}\text { Effect } \\
\text { Size }\end{array}$ & $\begin{array}{l}\mathrm{t}, \mathrm{z} \text {, or Chi- } \\
\text { square }\end{array}$ & $\begin{array}{l}P \\
\text { Value }\end{array}$ \\
\hline HAMD anxiety subscale & $5.8 \pm 2.2$ & $5.8 \pm 1.8$ & 0.000 & -0.129 a & 0.897 \\
\hline HAMD weight subscale & $1.0(0.0,2.0)$ & $0.0(0.0,1.0)$ & & $-1.757^{d}$ & 0.080 \\
\hline HAMD cognition subscale & $3.0 \pm 1.9$ & $3.0 \pm 1.6$ & 0.000 & $-0.073^{a}$ & 0.942 \\
\hline HAMD retard subscale & $6.8 \pm 1.7$ & $6.7 \pm 1.7$ & 0.059 & $-0.675^{a}$ & 0.500 \\
\hline HAMA somatic anxiety & $13.4 \pm 4.1$ & $13.3 \pm 3.6$ & 0.026 & $-0.133^{a}$ & 0.894 \\
\hline $\begin{array}{l}\text { HAMA psychological } \\
\text { anxiety }\end{array}$ & $6.9 \pm 4.3$ & $7.2 \pm 3.8$ & 0.066 & $0.615^{a}$ & 0.539 \\
\hline \multicolumn{6}{|c|}{$\begin{array}{l}\text { G1 = Group 1, patients with serious sleep symptoms; G2 = Group 2, patients without serious sleep } \\
\text { symptoms. }\end{array}$} \\
\hline \multicolumn{6}{|c|}{${ }^{\mathrm{a}}$ Independent sample t-test; ${ }^{\mathrm{b}}$ chi-square test; ${ }^{\mathrm{c}}$ Fisher's exact test; ${ }^{\mathrm{d}}$ nonparametric test. } \\
\hline
\end{tabular}

Table 3

Cognitive impairment in patients before matching.

\begin{tabular}{|c|c|c|c|c|c|c|}
\hline Cognitive domains & $\begin{array}{l}\text { G1 (n, } \\
\%)\end{array}$ & $\mathrm{G} 2(\mathrm{n}, \%)$ & $\begin{array}{l}\text { Chi- } \\
\text { square }\end{array}$ & OR & $95 \% \mathrm{Cl}$ & $P$ \\
\hline Attention and alertness & $\begin{array}{l}62 \\
(51.2)\end{array}$ & $\begin{array}{l}72 \\
(41.1)\end{array}$ & 2.943 & 1.503 & $\begin{array}{l}0.943 \\
2.397\end{array}$ & 0.087 \\
\hline Learn & $\begin{array}{l}38 \\
(31.4)\end{array}$ & $\begin{array}{l}47 \\
(26.9)\end{array}$ & 0.723 & 1.247 & $\begin{array}{l}0.749 \\
2.074\end{array}$ & 0.396 \\
\hline Memory & $\begin{array}{l}38 \\
(31.4)\end{array}$ & $\begin{array}{l}38 \\
(21.7)\end{array}$ & 3.520 & 1.651 & $\begin{array}{l}0.976 \\
2.793\end{array}$ & 0.062 \\
\hline Executive function & $\begin{array}{l}39 \\
(32.2)\end{array}$ & $\begin{array}{l}36 \\
(20.6)\end{array}$ & 5.141 & 1.836 & $\begin{array}{l}1.082 \\
3.116\end{array}$ & 0.024 \\
\hline Executive shifting & $\begin{array}{l}67 \\
(55.4)\end{array}$ & $\begin{array}{l}70 \\
(40.0)\end{array}$ & 6.799 & 1.816 & $\begin{array}{l}1.164 \\
2.975\end{array}$ & 0.009 \\
\hline Executive inhibition & $\begin{array}{l}43 \\
(35.5)\end{array}$ & $\begin{array}{l}41 \\
(23.4)\end{array}$ & 5.160 & 1.802 & $\begin{array}{l}1.081 \\
3.003\end{array}$ & 0.024 \\
\hline Word fluency & $51(42.1)$ & $51(29.1)$ & 5.358 & 1.771 & $\begin{array}{l}1.089 \\
2.881\end{array}$ & 0.021 \\
\hline $\begin{array}{l}\text { Information processing } \\
\text { speed }\end{array}$ & $44(36.4)$ & $44(25.1)$ & 4.311 & 1.701 & $\begin{array}{l}1.028 \\
2.816\end{array}$ & 0.039 \\
\hline
\end{tabular}


Table 4

Cognitive impairment in patients after matching.

\begin{tabular}{|c|c|c|c|c|c|c|}
\hline Cognitive domains & $\begin{array}{l}\text { G1 (n, } \\
\%)\end{array}$ & G2 (n, \%) & $\begin{array}{l}\text { Chi- } \\
\text { square }\end{array}$ & OR & $95 \% \mathrm{Cl}$ & $P$ \\
\hline Attention and alertness & $\begin{array}{l}62 \\
(51.2)\end{array}$ & $\begin{array}{l}47 \\
(38.8)\end{array}$ & 3.756 & 1.655 & $\begin{array}{l}0.993 \\
2.757\end{array}$ & 0.053 \\
\hline Learn & $\begin{array}{l}38 \\
(31.4)\end{array}$ & $\begin{array}{l}32 \\
(26.4)\end{array}$ & 0.724 & 1.221 & $\begin{array}{l}0.701 \\
2.126\end{array}$ & 0.481 \\
\hline Memory & $\begin{array}{l}38 \\
(31.4)\end{array}$ & $\begin{array}{l}29 \\
(24.0)\end{array}$ & 1.672 & 1.758 & $\begin{array}{l}0.980 \\
3.153\end{array}$ & 0.058 \\
\hline Executive function & $\begin{array}{l}39 \\
(32.2)\end{array}$ & $\begin{array}{l}23 \\
(19.0)\end{array}$ & 5.551 & 1.922 & $\begin{array}{l}1.068 \\
3.459\end{array}$ & 0.029 \\
\hline Executive shifting & $\begin{array}{l}67 \\
(55.4)\end{array}$ & $\begin{array}{l}48 \\
(39.7)\end{array}$ & 5.982 & 2.023 & $\begin{array}{l}\text { 1.211, } \\
3.379\end{array}$ & 0.007 \\
\hline Executive inhibition & $\begin{array}{l}43 \\
(35.5)\end{array}$ & $\begin{array}{l}27 \\
(22.3)\end{array}$ & 5.146 & 1.749 & $\begin{array}{l}1.000 \\
3.060\end{array}$ & 0.050 \\
\hline Word fluency & $\begin{array}{l}51 \\
(42.1)\end{array}$ & $\begin{array}{l}38 \\
(31.4)\end{array}$ & 3.003 & 1.591 & $\begin{array}{l}0.940 \\
2.695\end{array}$ & 0.084 \\
\hline $\begin{array}{l}\text { Information processing } \\
\text { speed }\end{array}$ & $\begin{array}{l}44 \\
(36.4)\end{array}$ & $\begin{array}{l}29 \\
(24.0)\end{array}$ & 4.414 & 1.813 & $\begin{array}{l}1.038 \\
3.167\end{array}$ & 0.037 \\
\hline
\end{tabular}

Compared with the patients in Group 2 (no serious sleep symptoms), chi-square analysis revealed that patients in Group 1 (symptoms of seriously disordered sleep) had more impairment in executive functioning ( $32.2 \%$ vs $19.0 \% ; P=0.029)$, executive shifting ( $55.4 \%$ vs $39.7 \% ; P=0.007)$, executive inhibition (35.5\% vs $22.3 \%, P=0.050)$, and information processing speed ( $36.4 \%$ vs $24.0 \% ; P=0.037$ ), respectively. After propensity score matching, for Group 1, the odds ratios for having a greater degree of cognitive impairment in executive functioning, executive shifting, executive inhibition, and information processing speed were 1.922 (95\% Cl: 1.068-3.459, $P=0.029)$, 2.023 (95\% Cl: 1.211-3.379, $P=0.007$ ), 1.749 (95\% Cl: $1.000-3.060, P=0.050)$, and 1.813 (95\% Cl: $1.038-3.167, P=0.037)$, respectively (Tables 3 and 4 ).

\section{Subgroup and Sensitivity Analyses}

We analyzed the effects of sleep disorder symptoms in Group 1 by age, sex, and depression severity in the domains of attention, learning, memory, and word fluency (Table 5). In the attention and alertness domain, the cognitive impairments were most obvious in patients aged 30 to 45 years $(\mathrm{OR}=2.511,95 \% \mathrm{Cl}$ : $1.153-5.468, P=0.020)$, in women $(\mathrm{OR}=2.129,95 \% \mathrm{Cl}: 1.150-3.943, P=0.016)$, and in patients with HAMD-17 scores $\leq 24(\mathrm{OR}=2.652,95 \% \mathrm{Cl}: 1.437-4.894, P=0.002)$ in each category. 
In the learning domain, the risk of cognitive impairment was the greatest in patients aged 30 to 45 years $(\mathrm{OR}=2.444,1.016-5.879, P=0.046)$.

In the memory domain, the risk was the highest in patients aged 30 to 45 years (OR $=4.345,95 \%$ Cl:1.667-11.326, $P=0.003)$, in women ( $\mathrm{OR}=2.318,95 \% \mathrm{Cl}: 1.106-4.858, P=0.026)$, and in patients with HAMD-17 scores $\leq 24$ (OR $=2.499,95 \% \mathrm{Cl}: 1.274-4.901, P=0.008)$ in each category.

Word fluency was most affected in patients aged $<30$ years $(\mathrm{OR}=4.400,95 \% \mathrm{Cl}: 1.337-14.483, P=$ 0.015).

We performed additional subgroup analyses in Group 1 of the effects of sleep disorders on executive functioning, executive shifting, executive inhibition, and the speed of information processing (Table 5). For executive functioning, the risk was the highest in patients aged 30 to 45 years (OR $=3.450,95 \%$ $\mathrm{Cl}: 1.367-8.704, P=0.009)$, in women (OR $=2.135,95 \% \mathrm{Cl}: 1.031-4.420, P=0.041)$, and in patients with HAMD-17 scores $\leq 24(\mathrm{OR}=2.232,95 \% \mathrm{Cl}: 1.132-4.398, P=0.020)$ in each category.

For executive shifting, the effects were the most pronounced in patients aged $<30$ years $(O R=13.714$, 95\% Cl:2.739-68.678, $P=0.001)$, in females (OR $=2.358,95 \% \mathrm{Cl}: 1.274-4.365, P=0.006)$, and in patients with HAMD-17 scores $>24(\mathrm{OR}=4.941,95 \% \mathrm{Cl}: 1.521-16.047, P=0.008)$ in each category.

For executive inhibition, the effects were the greatest in patients aged 30 to 45 years $(\mathrm{OR}=2.568,95 \% \mathrm{Cl}$ : $1.039-6.344, P=0.041)$.

Speed of information processing was the most affected in patients aged 30 to 45 years ( $O R=2.640,95 \%$ Cl: $1.101-6.328, P=0.030)$, in men $(\mathrm{OR}=2.976,95 \% \mathrm{Cl}: 1.122-7.896, P=0.028)$, and in patients with HAMD-17 scores $\leq 24(\mathrm{OR}=2.336,95 \% \mathrm{Cl}: 1.217-4.486, P=0.011)$ in each category.

\section{Discussion}

In this study, we evaluated the effects of seriously disordered sleep on cognitive functioning in treatmentnaïve patients with first-episode MDD and found a link between serious sleep disorders and impaired executive functioning and information processing speed. We found significant differences in stratification by sex, age, and depression severity between Groups 1 and 2 .

The baseline characteristics of the 2 groups had significant heterogeneity, so we performed propensity score matching to reduce bias, which reduced the sample size to 242 participants and enabled us to perform between-group comparisons, though the statistical power was decreased because the sample was somewhat smaller. There was a difference in the effect size of age between the 2 groups, though we had already considered the effect of age when we calculated the global deficit scores.

Previous studies have demonstrated that disordered sleep can affect cognitive functioning in various groups of people [33,34]. Moreover, several types of sleep disturbances have been shown to affect cognitive functioning. Some longitudinal studies found that insomnia is a risk factor for mild cognitive 
impairment and dementia [13, 21, 35]. In patients with multiple sclerosis, sleep disorders have been shown to impair cognitive functioning, especially in executive functioning [36]. Conversely, other studies have not found any relationship between sleep disorders and cognitive impairment. Astudy of 50 patients with chronic fatigue syndrome and 50 healthy controls found impaired executive functioning, but it was not related to sleep difficulties [37].

In our study, sleep disorders impaired cognition in the domains of executive functioning and information processing speed in patients with MDD. Other studies of patients with MDD have reported that sleep disturbances affect different cognitive domains. One study of 34 patients with MDD patients and 29 healthy controls found that poor sleep is an independent predictor of impaired executive functioning and especially visual-motor processing speed in patients with MDD [34]. A meta-analysis concluded that insomnia may affect a wide range of cognitive domains, including episodic memory, alertness and complex attention, manipulation in working memory, problem-solving, and perceptual functions [15]. The affected cognitive domains were different from those in our study, as were the methods for testing cognitive functioning.

Previous studies have explored the mechanisms underlying cognitive impairment in patients with MDD. Researchers have shown that sleep can affect both cognitive functioning and emotional processingby altering neural networks and decreasing brain-derived neurotrophic factor (BDNF); [38-40]. Executive functioning has been identified as one of the main impaired cognitive domains in patients with MDD, and impaired cognition has been suggested as one of the main barriers to functional recovery in these patients [1, 41]. Information processing speed has also consistently been found to be impaired in patients with MDD [1, 42]. Neural networks, including the prefrontal cortex, cingulate gyrus, subcortical regions in the striatum and thalamus, and temporal lobe structures, including the amygdala and hippocampus, have been found to be functionally altered in patients in a depressive state [43]. Deficits in executive functioning have been associated with pathophysiology in the lateral aspects of the prefrontal cortex. Functional alterations in the prefrontal cortex, cingulate gyrus, and subcortical regions, combined with deficits in executive functioning, have been observed in patients with sleep disorders [44]. The prefrontal cortex is the region responsible for executive functioning, and the subcortical regions are responsible for information processing speed. Another mechanism reportedly underlying cognitive impairment secondary to disordered sleep is related to the decrease of BDNF. One study conducted in a Chinese population found a lower level of BDNF in patients with clinical insomnia and cognitive impairment. Patients with MDD who had lower BDNF levels in the central nervous system had evidence of neural death and decreased brain volume, which led to cognitive impairment $[45,46]$. Rahmani and colleagues concluded that insomnia and depression arelinked to the down-regulation of hippocampal BDNF along with disrupted BDNF expression in the prefrontal cortex [47].

We stratified study participants by age, sex, and severity of MDD and analyzed the effects of sleep disturbances in different cognitive domains. Our results showed that patients who fell into discrete categories of age, sex, and depression severity were more impaired by sleep disorders. Specifically, we found that patients between the ages of 30 and 45 were more likely to have deficits in the domains of 
attention, learning, memory, executive functioning, and information processing speed. Patients younger than 30 years were more likely to experience cognitive impairment in the domains of executive shifting and word fluency. The correlation between sleep disturbances and impairment in word fluency domain was not significant after we performed propensity score matching, because the matching process eliminated some younger participants who did not have serious sleep disorders. We did observe a difference by sex. Sleep disorders were more likely to affect women in the domains of attention, memory, and executive functioning (especially executive shifting). For men, sleep disorders were more likely to affect information processing speed.

Cognitive impairment has also been reported to be related to MDD severity. Patients with mild to moderate depression have been shown to be more likely to experience cognitive impairment in the domains of attention, memory, executive functioning, and information processing speed. A sensitivity analysis conducted as part of a meta-analysis summarizing cognitive impairment in first-episode MDD patients concluded that neither age nor severity of depression was related to impaired cognition in the domains of attention, learning, memory, executive functioning, or information processing speed. These results are different from our findings, which may reflect the fact that the study participants included patients treated with medication, and that they were compared with normal controls [5].

Our study had some limitations. The cross-sectional study data we accessed had a weak cause-andeffect relationship. Future longitudinal studies will be essential in evaluating the link between sleep disturbance remission and cognitive impairment in MDD patients. Our sample population was restricted to Chinese adults aged 18 to 65 years, so our results may not be generalizable to other populations.Because our sample size was so small, we were not able to include some variables that can be risk factors for cognitive impairment in people with MDD. Also, the ages of the participants in our study varied so widely that a between-group imbalance in age ranges remained after propensity score matching. Finally, we used the HAMD sleep subscale to estimate sleep disorder severity. More specific measurement tools will be necessary to evaluate the effects of the stages of sleep in patients with MDD.

\section{Conclusions}

We explored the link between symptoms of disordered sleep and cognitive impairment in first-episode, treatment naïve patients with MDD,and stratified the effect of sleep disturbance symptoms by age,sex, and depression severity in different domains.To evaluate the presence and degree of cognitive impairment, we used a battery of comprehensive, cognitive measurement tests, which have proved sensitive in identifying impaired cognition in mental disorders.

Our results indicate that sleep disorder is associated with impaired executive functioning and delayed information processing speed in first-episode, treatment-naïve patients with MDD.Certain patients, including women between the ages of 30 and 45 years or patients with moderate depression, may be more likely to exhibit the symptoms of cognitive impairment.Sleep disorders can be important symptoms for physicians in clinical practice and can aid in recognizing cognitive impairment in patients with MDD. 
Therefore, sleep problems should be considered in depression management. Future longitudinal studies are warranted to confirm the effects of sleep disorders on cognitive functioning in patients with MDD.

\section{List Of Abbreviations}

MDD: major depressive disorder

MINI: Mini-International Neuropsychiatric Interview

HAMD-17: 17-item Hamilton Rating Scale for Depression

HAMA: Hamilton Anxiety Rating Scale

CPT: Continuous Performance Test

AVFS: Animal Verbal Fluency Scale

DSCT: Digit Symbol Coding Test

CTT: Color Trial Test

BVMT-R: Brief Visual Memory Test-Revised

HVLT-R: Hopkins Verbal Learning Test-Revised

SCWT: Stroop Color Word Test

MATRICS : the Measurement and Treatment Research to Improve Cognition in Schizophrenia MCCB : MATRICS Consensus Cognitive Battery

GDS: global deficit score

BMI: body mass index

FBG: fasting blood glucose

TC:total cholesterol

TG: triglycerides

LDL:Iow-density lipoprotein

CTQ: childhood trauma questionnaire

\section{Declarations}




\section{Ethics approval and consent to participate}

Written informed consent was obtained from all eligible participants. The study was approved by the research ethics board of the each institution including Peking University Sixth Hospital, Peking University Institute of Mental Health; Nankai University Affiliated Anding Hospital, Tianjin Mental Health Center; Peking Union Medical College Hospital; First Hospital of Shanxi Medical University; The First Affiliated Hospital of China Medical University; Dalian Seventh People's Hospital; Beijing Anding Hospital, Capital Medical University; The First Hospital of Hebei Medical University; and Tianjin Medical University General Hospital.

\section{Consent for publication}

not applicable.

\section{Availability of data and materials}

The dataset analyzed during the current study are available from the corresponding author on reasonable request.

\section{Competing Interest}

The authors declare that they have no competing interests.

\section{Funding}

The National Key Basic Research Program of China (NO. 2013CB531300), and National Science and Technology Major Project for Investigational New Drugs (NO. 2018ZX09201-014)provided financial support for this study. They had no influence on the conduction of the study, analysis of the data, or reporting of the results.

\section{Authors' contributions}

First author Feihuan Cui compiled the database, analyzed the data, wrote the manuscript, and made revisions as suggested by the reviewers.

Qi Liu, the corresponding author, was the study's principal investigator and directed most of the research.

Rainer Leonhart contributed to the statistical analysis.

Xiaozhen Lv,Hongjun Tian, Jing Wei, Kerang Zhang, Gang Zhu, Qiaoling Chen, Gang Wang, Xueyi Wang, and Nan Zhang were the principal investigators at the individual participating research centers and were responsible for directing and overseeing the research at their individual centers and ensuring the integrity of the results.

Yu Huang designed the database for this study. 
Tianmei Si and Xin Yu designed the study and registered the project, and had oversight over all research activities as the principal investigators of this multicenter study.

All authors approved the final version to be published.

All persons who have made substantial contributions to the work reported in this manuscript, including those who provided editing and writing assistance but who are not authors, are named in the acknowledgement section of the manuscript and have given their permission to be named.

\section{Acknowledgments}

The authors thank all participants in the study, as well as the investigators and sites involved in conducting the trial. The authors appreciate the help from Shuzhe Zhou, Xikun Han, and Zhiwei Jian during data analysis processing. We also would like to thank TopEdit (www.topeditsci.com) as well for English language editing of this manuscript.

\section{References}

1. Zuckerman H, Pan Z, Park C, Brietzke E, Musial N, Shariq AS, lacobucci M, Yim SJ, Lui L, Rong C et al: Recognition and Treatment of Cognitive Dysfunction in Major Depressive Disorder. FRONT PSYCHIATRY 2018, 9:655.

2. Clark M, DiBenedetti $D$, Perez V: Cognitive dysfunction and work productivity in major depressive disorder. Expert Rev Pharmacoecon Outcomes Res 2016, 16(4):455-463.

3. Carvalho AF, Miskowiak KK, Hyphantis TN, Kohler CA, Alves GS, Bortolato B, G SP, Machado-Vieira R, Berk M, McIntyre RS: Cognitive dysfunction in depression - pathophysiology and novel targets. CNS Neurol Disord Drug Targets 2014, 13(10):1819-1835.

4. Conradi HJ, Ormel J, de Jonge P: Presence of individual (residual) symptoms during depressive episodes and periods of remission: a 3-year prospective study. PSYCHOL MED 2011, 41(6):11651174.

5. Ahern E, Semkovska M: Cognitive functioning in the firstepisode of major depressive disorder: A systematic review and meta-analysis. NEUROPSYCHOLOGY 2017, 31(1):52-72.

6. Reppermund S, Ising M, Lucae S, Zihl J: Cognitive impairment in unipolar depression is persistent and non-specific: further evidence for the final common pathway disorder hypothesis. $P S Y C H O L$ MED 2009, 39(4):603-614.

7. Simons CJ, Jacobs N, Derom C, Thiery E, Jolles J, van Os J, Krabbendam L: Cognition as predictor of current and follow-up depressive symptoms in the general population. Acta Psychiatr Scand 2009, 120(1):45-52.

8. Lam RW, Mclntosh D, Wang J, Enns MW, Kolivakis T, Michalak EE, Sareen J, Song WY, Kennedy SH, MacQueen GM et al: Canadian Network for Mood and Anxiety Treatments (CANMAT) 2016 Clinical 
Guidelines for the Management of Adults with Major Depressive Disorder: Section 1. Disease Burden and Principles of Care. Can J Psychiatry 2016, 61(9):510-523.

9. Knight MJ, Air T, Baune BT: The role of cognitive impairment in psychosocial functioning in remitted depression. J Affect Disord 2018, 235:129-134.

10. Stickley A, Leinsalu M, DeVylder JE, Inoue Y, Koyanagi A: Sleep problems and depression among 237 023 community-dwelling adults in 46 low- and middle-income countries. Sci Rep 2019, 9(1):12011.

11. Boland EM, Vittengl JR, Clark LA, Thase ME, Jarrett RB: Is sleep disturbance linked to short- and longterm outcomes following treatments for recurrent depression? J Affect Disord 2020, 262:323-332.

12. Goldstone A, Javitz HS, Claudatos SA, Buysse DJ, Hasler BP, de Zambotti M, Clark DB, Franzen PL, Prouty DE, Colrain IM et al: Sleep Disturbance Predicts Depression Symptoms in Early Adolescence: Initial Findings From the Adolescent Brain Cognitive Development Study. J Adolesc Health 2020.

13. Ling A, Lim ML, Gwee X, Ho RC, Collinson SL, Ng TP: Insomnia and daytime neuropsychological test performance in older adults. SLEEP MED 2016, 17:7-12.

14. Li Y, Liu H, Weed JG, Ren R, Sun Y, Tan L, Tang X: Deficits in attention performance are associated with insufficiency of slow-wave sleep in insomnia. SLEEP MED 2016, 24:124-130.

15. Wardle-Pinkston S, Slavish DC, Taylor DJ: Insomnia and cognitive performance: A systematic review and meta-analysis. SLEEP MED REV 2019, 48:101205.

16. Thomas AJ, Gallagher P, Robinson LJ, Porter RJ, Young AH, Ferrier IN, O'Brien JT: A comparison of neurocognitive impairment in younger and older adults with major depression. PSYCHOL MED 2009, 39(5):725-733.

17. van Agtmaal M, Houben A, Pouwer F, Stehouwer C, Schram MT: Association of Microvascular Dysfunction With Late-Life Depression: A Systematic Review and Meta-analysis. JAMA PSYCHIAT 2017, 74(7):729-739.

18. Lemos-Miller A, Kearney CA: Depression and ethnicity as intermediary variables among dissociation, trauma-related cognitions, and PTSD symptomatology in youths. J NERV MENT DIS 2006, 194(8):584-590.

19. Dannehl K, Rief W, Euteneuer F: Childhood adversity and cognitive functioning in patients with major depression. Child Abuse Neg/ 2017, 70:247-254.

20. Withall A, Harris LM, Cumming SR: A longitudinal study of cognitive function in melancholic and non-melancholic subtypes of major depressive disorder. J Affect Disord 2010, 123(1-3):150-157.

21. Zimmerman ME, Bigal ME, Katz MJ, Brickman AM, Lipton RB: Sleep onset/maintenance difficulties and cognitive function in nondemented older adults: the role of cognitive reserve. $J$ Int Neuropsychol Soc 2012, 18(3):461-470.

22. Rosenstrom T, Jokela M, Puttonen S, Hintsanen M, Pulkki-Raback L, Viikari JS, Raitakari OT, Keltikangas-Jarvinen L: Pairwise measures of causal direction in the epidemiology of sleep problems and depression. PLOS ONE 2012, 7(11):e50841. 
23. Bagherzadeh-Azbari S, Khazaie H, Zarei M, Spiegelhalder K, Walter M, Leerssen J, Van Someren E, Sepehry AA, Tahmasian M: Neuroimaging insights into the link between depression and Insomnia: A systematic review. J Affect Disord 2019, 258:133-143.

24. HAMILTON M: A rating scale for depression. Journal of neurology, neurosurgery, and psychiatry 1960, 23(1):56-62.

25. Lv X, Si T, Wang G, Wang H, Liu Q, Hu C, Wang J, Su Y, Huang Y, Jiang H et al: The establishment of the objective diagnostic markers and personalized medical intervention in patients with major depressive disorder: rationale and protocol. BMC PSYCHIATRY 2016, 16:240.

26. Sheehan DV, Lecrubier Y, Sheehan KH, Amorim P, Janavs J, Weiller E, Hergueta T, Baker R, Dunbar GC: The Mini-International Neuropsychiatric Interview (M.I.N.I.): the development and validation of a structured diagnostic psychiatric interview for DSM-IV and ICD-10. J Clin Psychiatry 1998, 59 Suppl 20:22-33, 34-57.

27. Wang XD, Wang XL, Ma H: Handbook of Mental Health Assessment Scale. Beijing: Journal of Chinese Mental Health; 1999.

28. Wang XD, Wang. X L, Ma H: Handbook of Mental Health Assessment Scale. Beijing: Journal of Chinese Mental Health; 1999.

29. HAMILTON M: The assessment of anxiety states by rating. The British journal of medical psychology 1959, 32(1):50-55.

30. Yu X: Handbook of MATRICS Consensus Cognitive Battery Chinese Norm. Beijing: Peking

University Medical Press; 2014.

31. Carey CL, Woods SP, Gonzalez R, Conover E, Marcotte TD, Grant I, Heaton RK, HNRC G: Predictive validity of global deficit scores in detecting neuropsychological impairment in HIV infection. J CLIN EXP NEUROPSYC 2004, 26(3):307-319.

32. Bernstein DP, Fink L, Handelsman L, Foote J, Lovejoy M, Wenzel K, Sapareto E, Ruggiero J: Initial reliability and validity of a new retrospective measure of child abuse and neglect. The American journal of psychiatry 1994, 151(8):1132-1136.

33. Fortier-Brochu E, Morin CM: Cognitive impairment in individuals with insomnia: clinical significance and correlates. SLEEP 2014, 37(11):1787-1798.

34. Cabanel N, Schmidt AM, Fockenberg S, Bruckmann KF, Haag A, Muller MJ, Kundermann B: Evening preference and poor sleep independently affect attentional-executive functions in patients with depression. Psychiatry Res 2019, 281:112533.

35. McKinnon AC, Beath AP, Naismith SL: Relationships between sleep quality, depressive symptoms and MCl diagnosis: A path analysis. J Affect Disord 2019, 256:26-32.

36. Hughes AJ, Parmenter BA, Haselkorn JK, Lovera JF, Bourdette D, Boudreau E, Cameron MH, Turner AP: Sleep and its associations with perceived and objective cognitive impairment in individuals with multiple sclerosis. J SLEEP RES 2017, 26(4):428-435. 
37. Cockshell SJ, Mathias $\mathrm{JL}$ : Cognitive deficits in chronic fatigue syndrome and their relationship to psychological status, symptomatology, and everyday functioning. NEUROPSYCHOLOGY2013, 27(2):230-242.

38. Yin $H$, Zhang $L$, Li D, Xiao $L$, Cheng M: The gray matter volume of the right insula mediates the relationship between symptoms of depression/anxiety and sleep quality among college students. $J$ HEALTH PSYCHOL 2019:1573756441.

39. Wu CR, Chen PY, Hsieh SH, Huang HC, Chen YT, Chen TJ, Chiu HY: Sleep Mediates the Relationship Between Depression and Cognitive Impairment in Older Men. AM J MENS HEALTH2019, 13(1):1818103653.

40. Asarnow LD: Depression and sleep: what has the treatment research revealed and could the HPA axis be a potential mechanism? Curr Opin Psychol 2019, 34:112-116.

41. Cha DS, Carmona NE, Subramaniapillai M, Mansur RB, Lee Y, Hon Lee J, Lee J, Rosenblat JD, Shekotikhina M, Park C et al: Cognitive impairment as measured by the THINC-integrated tool (THINC-it): Association with psychosocial function in major depressive disorder. J AFFECT DISORDERS 2017, 222:14-20.

42. Liu J, Dong Q, Lu X, Sun J, Zhang L, Wang M, Wan P, Guo H, Zhao F, Ju Y et al: Exploration of Major Cognitive Deficits in Medication-Free Patients With Major Depressive Disorder. FRONT PSYCHIATRY 2019, 10:836.

43. Clark L, Chamberlain SR, Sahakian BJ: Neurocognitive mechanisms in depression: implications for treatment. ANNU REV NEUROSCI 2009, 32:57-74.

44. Grau-Rivera O, Operto G, Falcon C, Sanchez-Benavides G, Cacciaglia R, Brugulat-Serrat A, Gramunt N, Salvado G, Suarez-Calvet M, Minguillon $\mathrm{C}$ et al: Association between insomnia and cognitive performance, gray matter volume, and white matter microstructure in cognitively unimpaired adults. ALZHEIMERS RES THER 2020, 12(1):4.

45. Fan TT, Chen WH, Shi L, Lin X, Tabarak S, Chen SJ, Que JY, Bao YP, Tang XD, Shi J et al: Objective sleep duration is associated with cognitive deficits in primary insomnia: BDNF may play a role. SLEEP 2019, 42(1).

46. Kim HK, Nunes PV, Oliveira KC, Young LT, Lafer B: Neuropathological relationship between major depression and dementia: A hypothetical model and review. Prog Neuropsychopharmacol Biol Psychiatry 2016, 67:51-57.

47. Rahmani M, Rahmani F, Rezaei N: The Brain-Derived Neurotrophic Factor: Missing Link Between Sleep Deprivation, Insomnia, and Depression. NEUROCHEM RES 2020, 45(2):221-231.

\section{Tables}

Table 5. Subgroup analysis of cognitive domains in patients after propensity score matching. 


\begin{tabular}{|c|c|c|c|c|c|}
\hline Attention andalertness & G1 $(n, \%)$ & $\mathrm{G} 2(\mathrm{n}, \%)$ & OR & $95 \% \mathrm{Cl}$ & $P$ \\
\hline age $<30$ & $11(61.1)$ & $17(44.7)$ & 1.941 & $0.619,6.089$ & 0.255 \\
\hline$\geq 30,<45$ & $30(53.6)$ & $17(31.5)$ & 2.511 & $1.153,5.468$ & 0.020 \\
\hline$\geq 45$ & $21(44.7)$ & $13(44.8)$ & 0.994 & $0.392,2.521$ & 0.990 \\
\hline male & $18(46.2)$ & $16(48.5)$ & 0.844 & $0.360,2.305$ & 0.844 \\
\hline female & $44(53.7)$ & $31(35.2)$ & 2.129 & $1.150,3.943$ & 0.016 \\
\hline $\mathrm{HAMD} \leq 24$ & $46(60.5)$ & $37(36.6)$ & 2.652 & $1.437,4.894$ & 0.002 \\
\hline HAMD $₫ 24$ & $16(35.6)$ & $10(50.0)$ & 0.552 & $0.190,1.605$ & 0.552 \\
\hline Learn & G1 (n, \%) & $\mathrm{G} 2(\mathrm{n}, \%)$ & OR & $95 \% \mathrm{Cl}$ & $P$ \\
\hline age $<30$ & $6(33.3)$ & 15(39.5) & 0.767 & $0.236,2.486$ & 0.658 \\
\hline$\geq 30,<45$ & $20(35.7)$ & 10(18.5) & 2.444 & $1.016,5.879$ & 0.046 \\
\hline$\geq 45$ & $12(25.5)$ & $8(27.6)$ & 0.900 & $0.316,2.560$ & 0.843 \\
\hline male & 14(35.9) & $14(42.4)$ & 0.760 & $0.294,1.967$ & 0.572 \\
\hline female & $24(29.3)$ & 19(21.6) & 1.503 & $0.749,3.013$ & 0.251 \\
\hline HAMD $\leq 24$ & $28(36.8)$ & $27(26.7)$ & 1.599 & $0.842,3.036$ & 0.152 \\
\hline HAMD $₫ 24$ & $10(22.2)$ & $6(30.0)$ & 0.667 & $0.203,2.184$ & 0.503 \\
\hline Memory & $\mathrm{G} 1(\mathrm{n}, \%)$ & $\mathrm{G} 2(\mathrm{n}, \%)$ & OR & $95 \% \mathrm{Cl}$ & $P$ \\
\hline age $<30$ & $4(22.2)$ & $9(23.7)$ & 0.921 & $0.241,3.514$ & 0.904 \\
\hline$\geq 30,<45$ & $22(39.3)$ & $7(13.0)$ & 4.345 & $1.667,11.326$ & 0.003 \\
\hline$\geq 45$ & $12(25.5)$ & $9(31.0)$ & 0.762 & $0.274,2.122$ & 0.603 \\
\hline male & 13(33.3) & 11(33.3) & 1.000 & $0.374,2.674$ & 0.999 \\
\hline female & $25(30.5)$ & 14(15.9) & 2.318 & $1.106,4.858$ & 0.026 \\
\hline $\mathrm{HAMD} \leq 24$ & $29(38.2)$ & $20(19.8)$ & 2.499 & $1.274,4.901$ & 0.008 \\
\hline HAMD $₫ 24$ & $9(20.0)$ & $5(25.0)$ & 0.750 & $0.215,2.613$ & 0.651 \\
\hline Executive function & $\mathrm{G} 1(\mathrm{n}, \%)$ & $\mathrm{G} 2(\mathrm{n}, \%)$ & OR & $95 \% \mathrm{Cl}$ & $P$ \\
\hline age $<30$ & $8(44.4)$ & $9(23.7)$ & 2.578 & $0.782,8.500$ & 0.120 \\
\hline$\geq 30,<45$ & $21(37.5)$ & $8(14.8)$ & 3.450 & $1.367,8.704$ & 0.009 \\
\hline$\geq 45$ & $10(21.3)$ & $7(24.1)$ & 0.849 & $0.283,2.553$ & 0.771 \\
\hline
\end{tabular}




\begin{tabular}{|c|c|c|c|c|c|}
\hline male & $14(35.9)$ & $9(27.3)$ & 1.493 & $0.545,4.090$ & 0.435 \\
\hline female & $25(30.5)$ & 15(17.0) & 2.135 & $1.031,4.420$ & 0.041 \\
\hline $\mathrm{HAMD} \leq 24$ & $27(35.5)$ & 20(19.8) & 2.232 & $1.132,4.398$ & 0.020 \\
\hline HAMD®24 & $12(26.7)$ & $4(20.0)$ & 1.455 & $0.405,5.229$ & 0.566 \\
\hline Executiveshifting & G1 $(n, \%)$ & G2 $(n, \%)$ & OR & $95 \% \mathrm{Cl}$ & $P$ \\
\hline age $<30$ & 16(88.9) & $14(36.8)$ & 13.714 & $2.739,68.678$ & 0.001 \\
\hline$\geq 30,<45$ & $25(44.6)$ & 17(31.5) & 1.755 & $0.805,3.826$ & 0.157 \\
\hline$\geq 45$ & $26(55.3)$ & 15(51.7) & 1.156 & $0.457,2.923$ & 0.760 \\
\hline male & 18(46.2) & 12(36.4) & 1.500 & $0.581,3.872$ & 0.402 \\
\hline female & $49(59.8)$ & $34(38.6)$ & 2.358 & $1.274,4.365$ & 0.006 \\
\hline $\mathrm{HAMD} \leq 24$ & $39(51.3)$ & $41(40.6)$ & 1.543 & $0.847,2.811$ & 0.157 \\
\hline HAMD $₫ 24$ & $28(62.2)$ & $5(25.0)$ & 4.941 & $1.521,16.047$ & 0.008 \\
\hline Executive inhibition & G1 (n, \%) & $\mathrm{G} 2(\mathrm{n}, \%)$ & OR & $95 \% \mathrm{Cl}$ & $P$ \\
\hline age $<30$ & 10(55.6) & 13(34.2) & 2.404 & $0.764,7.562$ & 0.134 \\
\hline$\geq 30,<45$ & 19(33.9) & $9(16.7)$ & 2.568 & $1.039,6.344$ & 0.041 \\
\hline$\geq 45$ & 14(29.8) & $7(24.1)$ & 1.333 & $0.464,3.831$ & 0.593 \\
\hline male & $23(59.0)$ & $14(42.4)$ & 1.951 & $0.762,4.994$ & 0.164 \\
\hline female & $20(24.4)$ & 15(17.0) & 1.570 & $0.741,3.324$ & 0.239 \\
\hline HAMD $\leq 24$ & $29(38.2)$ & $25(24.8)$ & 1.876 & $0.982,3.582$ & 0.057 \\
\hline HAMD $₫ 24$ & 14(31.1) & $4(20.0)$ & 1.806 & $0.510,6.397$ & 0.359 \\
\hline Word fluency & G1 $(n, \%)$ & $\mathrm{G} 2(\mathrm{n}, \%)$ & OR & $95 \% \mathrm{Cl}$ & $P$ \\
\hline age $<30$ & 11(61.1) & $10(26.3)$ & 4.400 & $1.337,14.483$ & 0.015 \\
\hline$\geq 30,<45$ & $22(39.3)$ & 21(38.9) & 1.017 & $0.473,2.188$ & 0.996 \\
\hline$\geq 45$ & 18(38.3) & $7(24.1)$ & 1.951 & $0.694,5.486$ & 0.205 \\
\hline male & 14(35.9) & $9(27.3)$ & 1.493 & $0.545,4.090$ & 0.435 \\
\hline female & $37(45.1)$ & $29(33.0)$ & 1.673 & $0.898,3.116$ & 0.105 \\
\hline HAMD $\leq 24$ & $31(40.8)$ & 31(30.7) & 1.556 & $0.834,2.900$ & 0.164 \\
\hline HAMD $₫ 24$ & $20(44.4)$ & $7(35.0)$ & 1.486 & $0.499,4.423$ & 0.477 \\
\hline Information processing speed & $\mathrm{G} 1(\mathrm{n}, \%)$ & $\mathrm{G} 2(\mathrm{n}, \%)$ & OR & $95 \% \mathrm{Cl}$ & $P$ \\
\hline
\end{tabular}




\begin{tabular}{|llllll|} 
age $<30$ & $11(61.1)$ & $14(36.8)$ & 2.694 & $0.849,8.545$ & 0.092 \\
$\geq 30,<45$ & $21(37.5)$ & $10(18.5)$ & 2.640 & $1.101,6.328$ & 0.030 \\
$\geq 45$ & $12(25.5)$ & $5(17.2)$ & 1.646 & $0.513,5.278$ & 0.402 \\
male & $22(56.4)$ & $10(30.3)$ & 2.976 & $1.122,7.896$ & 0.028 \\
female & $22(26.8)$ & $19(21.6)$ & 1.332 & $0.658,2.694$ & 0.426 \\
\hline HAMD $\leq 24$ & $31(40.8)$ & $23(22.8)$ & 2.336 & $1.217,4.486$ & 0.011 \\
\hline HAMD $\otimes 24$ & $13(28.9)$ & $6(30.0)$ & 0.948 & $0.299,3.004$ & 0.928 \\
\hline
\end{tabular}

G1 = Group 1, patients with serious sleep symptoms (HAMD-17 sleep score $>4$ );G2 = Group 2, patients without serious sleep symptoms (HAMD-17 sleep score $\leq 4)$. 